\title{
PET/MRI for Oncologic Brain Imaging: A Comparison of Standard MR-Based Attenuation Corrections with a Model- Based Approach for the Siemens mMR PET/MR System
}

\author{
Ivo Rausch ${ }^{1}$, Lucas Rischka ${ }^{2}$, Claes N. Ladefoged ${ }^{3}$, Julia Furtner ${ }^{4}$, Matthias Fenchel ${ }^{5}$, Andreas Hahn², \\ Rupert Lanzenberger ${ }^{2}$, Marius E. Mayerhoefer ${ }^{4}$, Tatjana Traub-Weidinger ${ }^{6}$, and Thomas Beyer ${ }^{1}$ \\ ${ }^{1}$ Center for Medical Physics and Biomedical Engineering, Medical University of Vienna, Vienna, Austria; ${ }^{2}$ Department of Psychiatry \\ and Psychotherapy, Medical University of Vienna, Vienna, Austria; ${ }^{3}$ Department of Clinical Physiology, Nuclear Medicine and PET, \\ University of Copenhagen, Copenhagen, Denmark; ${ }^{4}$ Division of Neuroradiology, Department of Biomedical Imaging and Image- \\ Guided Therapy, Medical University of Vienna, Vienna, Austria; ${ }^{5}$ Siemens Healthcare GmbH, Magnetic Resonance, Erlangen, \\ Germany; and ${ }^{6}$ Division of Nuclear Medicine, Department of Biomedical Imaging and Image-guided Therapy, Medical University of \\ Vienna, Vienna, Austria
}

The aim of this study was to compare attenuation-correction (AC) approaches for PET/MRI in clinical neurooncology. Methods: Fortynine PET/MRI brain scans were included: brain tumor studies using ${ }^{18} \mathrm{~F}$-fluoro-ethyl-tyrosine $\left({ }^{18} \mathrm{~F}-\mathrm{FET}\right)(n=31)$ and ${ }^{68} \mathrm{Ga}-\mathrm{DOTANOC}$ $(n=7)$ and studies of healthy subjects using ${ }^{18} \mathrm{~F}-\mathrm{FDG}(n=11)$. For each subject, MR-based AC maps (MR-AC) were acquired using the standard DIXON- and ultrashort echo time (UTE)-based approaches. A third MR-AC was calculated using a model-based, postprocessing approach to account for bone attenuation values (BD, noncommercial prototype software by Siemens Healthcare). As a reference, AC maps were derived from patient-specific CT images (CTref). PET data were reconstructed using standard settings after $A C$ with all 4 AC methods. We report changes in diagnosis for all brain tumor patients and the following relative differences values (RDs [\%]), with regards to AC-CTref: for ${ }^{18} \mathrm{~F}-\mathrm{FET}(\mathrm{A})$ SUVs as well as volumes of interest (VOIs) defined by a $70 \%$ threshold of all segmented lesions and lesion-to-background ratios; for ${ }^{68} \mathrm{Ga}-\mathrm{DOTANOC}(\mathrm{B})$ - SUVs as well as VOls defined by a $50 \%$ threshold for all lesions and the pituitary gland; and for ${ }^{18} \mathrm{~F}-\mathrm{FDG}$ (C) - RD of SUVs of the whole brain and 10 anatomic regions segmented on MR images. Results: For brain tumor imaging ( $A$ and $B$ ), the standard PET-based diagnosis was not affected by any of the 3 MR-AC methods. For A, the average RDs of SUV mean were $-10 \%$, $-4 \%$, and $-3 \%$ and of the VOls $1 \%, 2 \%$, and $7 \%$ for DIXON, UTE, and $\mathrm{BD}$, respectively. Lesion-to-background ratios for all MR-AC methods were similar to that of CTref. For B, average RDs of SUV $_{\text {mean }}$ were $-11 \%,-11 \%$, and $-3 \%$ and of the VOIs $1 \%$, $-4 \%$, and $-3 \%$, respectively. In the case of ${ }^{18} \mathrm{~F}-\mathrm{FDG}$ PET/MRI (C), RDs for the whole brain were $-11 \%,-8 \%$, and $-5 \%$ for DIXON, UTE, and BD, respectively. Conclusion: The diagnostic reading of $P E T / M R$ patients with brain tumors did not change with the chosen AC method. Quantitative accuracy of SUVs was clinically acceptable for UTE- and BD-AC for group A, whereas for group B BD was in accordance with CTref. Nevertheless, for the quantification of individual lesions large deviations to CTref can be observed independent of the MR-AC method used.

Received Oct. 28, 2016; revision accepted Jan. 31, 2017.

For correspondence or reprints contact: Tatjana Traub-Weidinger, Waehringer Guertel 18-20/3L, 1090 Vienna, Austria.

E-mail: tatjana.traub-weidinger@meduniwien.ac.at

Published online Mar. 2, 2017.

COPYRIGHT (C) 2017 by the Society of Nuclear Medicine and Molecular Imaging.
Key Words: PET/MRI; brain tumor imaging; attenuation correction; quantification

J Nucl Med 2017; 58:1519-1525

DOI: 10.2967/jnumed.116.186148

M RI and PET are widely used imaging modalities in neurooncology $(1,2)$. Through the combination of PET and MRI into a single imaging system (PET/MRI) these 2 modalities have been joined into a promising new imaging tool (3-5). PET/MRI benefits from the high-resolution anatomic details provided by MRI and the high functional sensitivity by PET. Therefore, for neurologic examinations PET/MRI using task-specific tracers is under investigation (5). For example, ${ }^{18} \mathrm{~F}$-FDG is used in neurologic disorders, such as dementia, Parkinson, and epilepsy. Different ${ }^{18}$ F-FDG uptake patterns, especially within the cortex, are described for disease classification (6-8). Aminoacid tracers, such as ${ }^{18} \mathrm{~F}$-fluoro-ethyl-tyrosine $\left({ }^{18} \mathrm{~F}-\mathrm{FET}\right)$, are used for glioma imaging for prognosis, treatment planning, and treatment response assessment (2). Moreover, radiolabeled somatostatin analogs can be used with high sensitivity for meningioma imaging (9). Together with the anatomic details provided by MRI, PET/MRI seems to be beneficial for all of these tracers in comparison to independent PET and MRI examinations (4).

However, PET/MRI is a relatively new technique that faces several methodologic challenges (10), for example, MRI-based attenuation correction (MR-AC) (11). PET imaging relies on the detection of annihilation photons. These photons are attenuated by the traversed tissue, which leads to an underestimation of tracer uptake. Therefore, $\mathrm{AC}$ is needed, which was formerly done using a transmission scan with a rotating positron source in stand-alone PET systems (12) or a CT scan in combined PET/CT systems (13). The information gained from these scans can be used to derive the attenuation coefficients and correction factors of the PET data (14).

In contrast, MRI is based on the measurement of the magnetization of atomic nuclei, which is not related to electron densities that determine the attenuation of photons. Therefore, various MRAC methods have been developed that estimate photon attenuation properties from dedicated MRI sequence-based images (15). Such 
methods rely, for example, on segmentation approaches applied directly to the MR images (direct segmentation), a predefined template (template-based methods), or a combination of atlas/modelbased approaches (atlas-based methods) from which additional information from a database, typically based on conjoined CT and MR image data, is incorporated (16).

In the current fully integrated PET/MRI systems, 3 techniques are available for AC of brain acquisitions. To date, the Siemens Biograph mMR PET/MRI system (17) provides 2 MR-AC methods based on the direct segmentation of the MR image information. One is based on a DIXON VIBE (volume interpolated breath-hold examination) sequence resulting in an $\mathrm{AC}$ map, thereby dividing the measured subject into 4 discrete tissue classes: soft tissue, fat, lung, and air $(18,19)$. This method has a good reproducibility (19) but leads to systematic underestimations of attenuation coefficients in the presence of bony structures $(11,20)$. The second MR-AC method is based on an ultrashort echo time (UTE) sequence (21), primarily used for brain examinations. This method uses 3 discrete tissue classes, namely soft tissue, bone, and air. It improves quantification in comparison to DIXON MR-AC through the incorporation of bone information at the cost of an increased sensitivity to susceptibility artifacts (21). A third method is based on the incorporation of bone information from a model into the standard MR-AC. Such a method currently exists as a noncommercial prototype software from Siemens Healthcare; it adds bone attenuation to the 4-compartment attenuation maps based on DIXON input images $(22,23)$. All of these methods have been evaluated quantitatively for ${ }^{18} \mathrm{~F}-\mathrm{FDG}$ brain examinations $(21,23,24)$. The analyses in these studies were based on a global and regional comparison of tracer uptake between PET images reconstructed with the respective AC method and a CT-based AC approach. Nevertheless, little is known about the performance of these AC methods in neurooncologic studies and in clinical practice, including visual diagnosis, quantitative evaluations, and lesion contouring. Such knowledge is, however, required to further integrate PET/MRI into the clinical management of patients with neurooncologic diseases.

Therefore, the aim of this study was to evaluate PET/MRI examinations in clinical neurooncology imaging scenarios using the currently implemented MR-AC approaches in the Siemens Biograph mMR PET/MRI system, including a model-based AC approach in comparison to CT-based AC as the reference standard. This was done for non- ${ }^{18}$ F-FDG PET scans of patients with suspected brain lesions according to the standard clinical interpretation. Further, quantitative differences were assessed using lesion-defined delineations according to a possible clinical scenario. To enable a comparison with already-published data $(21,23,24)$, ${ }^{18} \mathrm{~F}-\mathrm{FDG}$ scans of healthy volunteers were analyzed also for global and regional quantitative differences.

\section{MATERIALS AND METHODS}

\section{Subjects and Acquisition}

This retrospective study consists of 49 subjects who underwent single-injection, dual-measurement protocols comprising a PET/MRI examination on a Biograph mMR PET/MRI system (Siemens Healthcare) and subsequent imaging on a Siemens Biograph True Point True View PET/CT system. Data were pooled from 2 studies, both approved by the local ethics committee, and subjects signed a written informed consent form. Subjects were arranged into patients with suspected tumors (groups A and B) and healthy volunteers (group C).
Group A. For brain tumor imaging, 31 patients, who were referred for an ${ }^{18} \mathrm{~F}$-FET PET examination for brain tumor assessment were included. The protocol consisted of a 40-min dynamic, single-bedposition PET scan for PET/MRI. For this study, PET data of the last $10 \mathrm{~min}$ of the 40-min scan (30-40 min) were extracted and used for image reconstruction and data analysis.

Group B. Seven patients with the diagnosis of meningioma, who underwent a ${ }^{68} \mathrm{Ga}$-DOTANOC PET/MRI examination, were included. The protocol consisted of a 10-min static PET examination starting at 40 min after injection.

Group $C$. For the ${ }^{18} \mathrm{~F}-\mathrm{FDG}$ imaging, 11 healthy subjects underwent a 95-min dynamic ${ }^{18} \mathrm{~F}-\mathrm{FDG}$ PET/MRI examination of the brain. For this study, a 10-min standard acquisition was extracted from the data starting at $50 \mathrm{~min}$ after injection.

All PET/MRI acquisitions included the sequences used for the derivation of standard MR-AC maps (DIXON and UTE) as well as an anatomic T1-weighted image (magnetization-prepared rapid gradient echo sequence with an echo time of $4.2 \mathrm{~ms}$, repetition time of 2,000 $\mathrm{ms}$, and voxel size of $1 \times 1 \times 1 \mathrm{~mm}$ ) for anatomic correlation of the tracer uptake.

After the PET/MRI examination, all subjects underwent a low-dose CT of the head. These CT data were used for CT-based AC for the purpose of this study.

\section{Data Processing}

DIXON and UTE sequences were acquired simultaneously with the PET emission data in all patients. For the model-based AC, the DIXON-based AC map and the underlying MR images (in-phase, opposed-phase, fat and water images) were used as an input for the model-based prototype segmentation algorithm (Siemens Healthcare $\mathrm{GmbH}$ ). The prototype approach automatically coregisters (nonrigidly) an MR image of a model consisting of a pair of MR and corresponding CT images to the MR images of the DIXON-based AC approach and inserts the corresponding attenuation coefficients of bone from the similar bone attenuation maps into the DIXON-based AC map. This results in a new AC map (BD) incorporating spatially variant information of bony tissue. Further information about the method can be found in other studies $(22,23)$.

For the CT-based AC, the low-dose CT was coregistered rigidly to the anatomic MR image after removal of the patient bed from the CT image. These coregistered images were then resampled to the pixel size of the original PET/MRI AC maps and scaled to linear attenuation values using the bilinear scaling (25) as implemented in the PET/CT (Hounsfield units $+1,000<1,070$ : slope $=9.5 \times 10^{-5}$, intercept $=0$; Hounsfield units $+1,000 \geq 1,070$ : slope $=5.1 \times 10^{-5}$, intercept $=$ $\left.4.7 \times 10^{-2}\right)$. Subsequently, the pixel data of the original DIXON-based AC map were exchanged with these data, resulting in a new AC map based on the original CT (CTref).

Reconstructions of all datasets were performed using the Siemens reconstruction software (e7tools). Image reconstruction was based on an ordinary Poisson ordered-subsets expectation maximization algorithm with 3 iterations and 21 subsets. A matrix size of $172 \times 172$ pixels with a zoom factor of 2 was used, thus resulting in a pixel size of $2 \times 2 \mathrm{~mm}$. For each patient, all $4 \mathrm{AC}$ maps were used during PET reconstruction, thus resulting in 4 PET images. The AC maps of the hardware components (i.e., patient table and head coil) consisting of CT-based templates implemented for the above PET/MR system were automatically added during reconstruction. In the case of groups $\mathrm{A}\left({ }^{18} \mathrm{~F}-\mathrm{FET}\right)$ and $\mathrm{B}\left({ }^{68} \mathrm{Ga}-\right.$ DOTANOC), a postreconstruction filter (a gaussian kernel of $3 \mathrm{~mm}$ in full width at half maximum) was applied to the images according to the onsite clinical routine for brain tumor imaging. Similarly, a $5-\mathrm{mm}$ gaussian filter was used for reconstruction in group C ( $\left.{ }^{18} \mathrm{~F}-\mathrm{FDG}\right)$. 


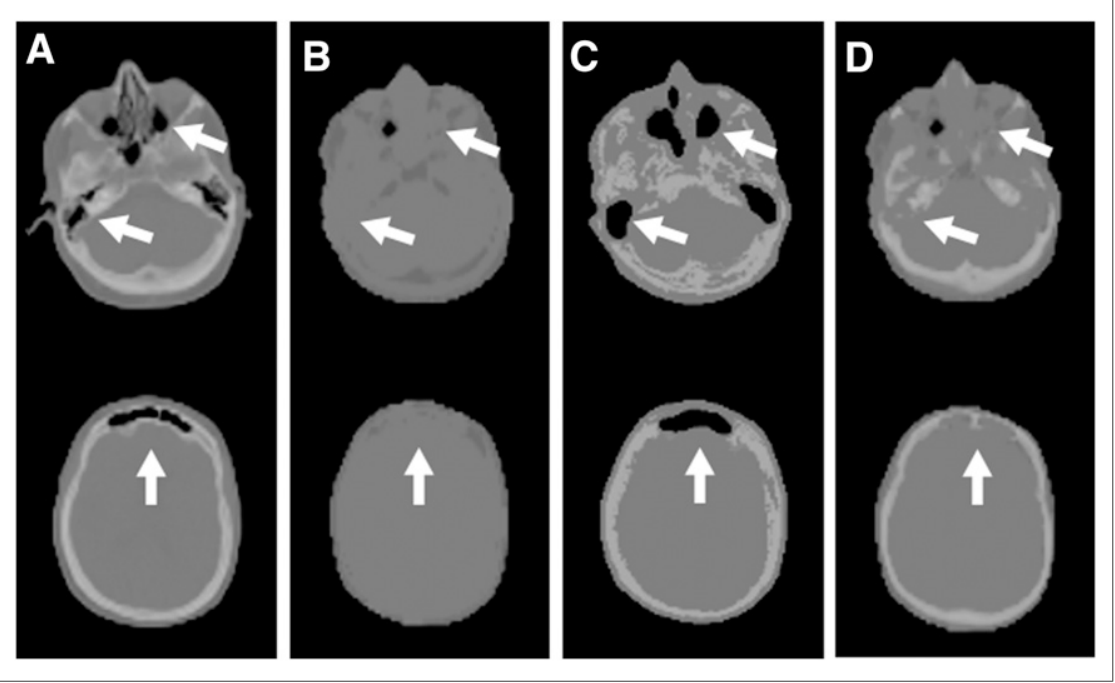

FIGURE 1. Example of artifact categories. Axial views at 2 main axial levels of same patient and different $A C$ methods: CT-reference (A), DIXON-artificial filling of air cavities with tissue (B), UTE-overestimation of air cavities due to susceptibility artifacts (C), and BD-based MR-ACtranslation of artifacts found in DIXON (D). Arrows indicate artifacts.

\section{Analysis}

For the brain tumor examinations, the DIXON- and UTE-based MR-AC maps were inspected visually for artifacts by comparing the MR-AC maps with the respective CTref. For the DIXON-based AC, the artifact categories contained misclassification of air by fatty or soft tissue in the sinuses, paranasal sinuses, and ears/mastoid cells. In the case of UTE, susceptibility artifacts (classifying bone as air or overestimating the extent of the air cavities) in these regions were assessed (Fig. 1). In the case of the BD, the assessment of artifacts was omitted because this MR-AC method is based on DIXON MR-AC, and therefore artifacts in the original MR-AC translate directly into BD-based MR-AC.

To evaluate differences in diagnostic outcome, PET images of all brain tumor patients (groups A and B) were assessed by a nuclear medicine specialist with $10 \mathrm{y}$ of experience in neurologic PET
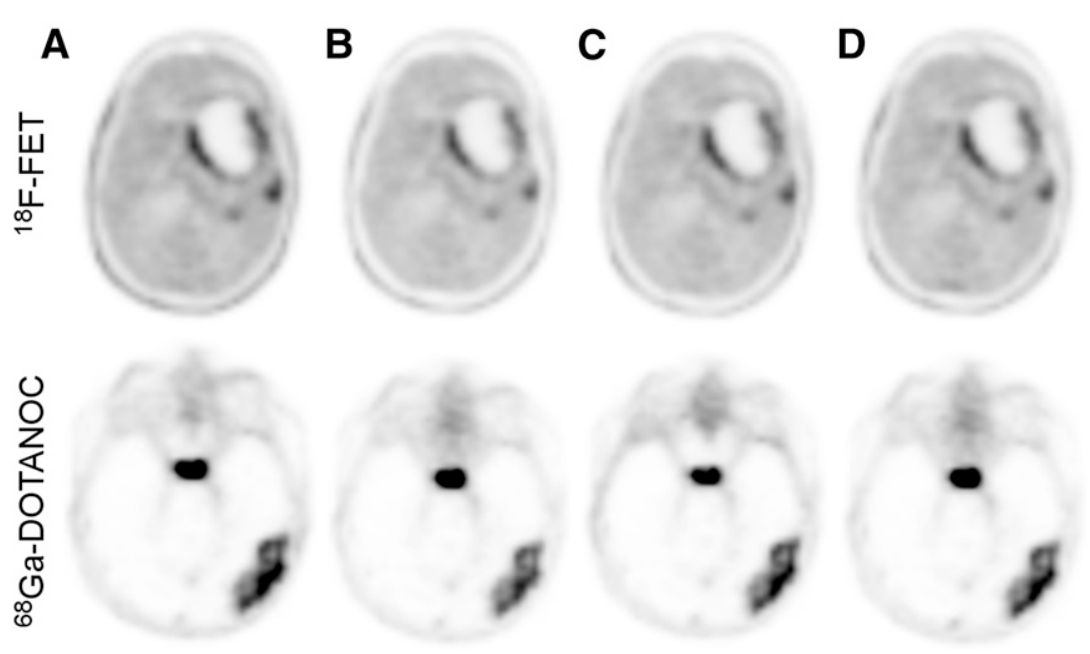

FIGURE 2. Axial views of patient with tumor recurrence imaged with ${ }^{18} \mathrm{~F}-\mathrm{FET}$ (top) and patient with a meningeal tumor formation imaged with ${ }^{68} \mathrm{Ga}-\mathrm{DOTANOC}$ (bottom). PET images were reconstructed after AC using: CTref-reference (A), DIXON (B), UTE (C), and BD (D). $\mathrm{SUV}_{\text {mean }}$ of the delineated $\mathrm{VOI}_{70} \mathrm{~S}\left(\mathrm{SUV}_{\text {mean }}\right)$. RDs were also calculated for the LBRs of the $\mathrm{SUV}_{\max }$ and $\mathrm{SUV}_{\text {mean }}$. For group $\mathrm{B}$, for ${ }^{68} \mathrm{Ga}-$ DOTANOC-avid lesions, RDs were calculated for VOIs defined by a $50 \%$ threshold $\left(\mathrm{VOI}_{50}\right)(27,28)$ and for the corresponding $\mathrm{SUV}_{\max }$ and $\mathrm{SUV}_{\text {mean }}$ for all lesions as well as the pituitary gland. The $\mathrm{VOI}_{50} \mathrm{~s}$ were further subcategorized in lesions attached to the skull base or the skull cap and analyzed separately. For group $\mathrm{C}$, for the ${ }^{18} \mathrm{~F}$-FDG imaging, average RD images of tracer uptake for DIXON-, UTE-, and BD- to CTref-based PET were produced. This was done after nonlinear alignment (ANTs; PICSL) of each individual T1-weighted image to Montreal Neurological Institute (MNI) space using the ICBM 2009a T1-weighted average structural template, and subsequently warping the PET image to MNI space before calculating the average RD in tracer uptake (24). Then, the wholebrain bias (mean of all voxels within the brain) of the tracer uptake was calculated for each of the 3 MR-AC methods. In addition, the RD in tracer uptake for 10 reference regions (capsula interna, cerebellum, frontal cortex, medulla, caudate nu-

imaging. Diagnostic reading was based on account lesion-to-background ratios (LBRs) for the ${ }^{18} \mathrm{~F}$-FET scans. The criteria for the ible lesions, their location, size, and shape as well as the tracer distribution in the lesion based on the clinical color scale using the
software Hybrid Viewer 3.6 (Hermes Medical Solutions). LBRs were calculated as the ratio of the SUVs of the maximum pixel in the background volume of interest (VOI) with a diameter of $3 \mathrm{~cm}$, which was placed on the contralateral side of the lesion.

tions with different MR-AC methods was done in terms of relative difference (RD) to the CTref-based findings. Threshold-based segmentations were used to avoid interreader ariability in delineating the brain lesions.

In accordance with the literature, for group A, a $70 \%$ threshold $\left(\mathrm{VOI}_{70}\right)$ of the maximum pixel was used for ${ }^{18} \mathrm{~F}$-FET-avid lesions $(26,27)$. RDs were calculated for the $\mathrm{VOI}_{70}, \mathrm{SUV}_{\mathrm{max}}$, and cleus, occipital, parietal, putamen, temporal, thalamus) within the brain was calculated (24). Further, each ${ }^{18} \mathrm{~F}$-FET-avid lesion was assigned to 1 of the 10 anatomic regions used for the regional analysis of the ${ }^{18} \mathrm{~F}-\mathrm{FDG}$ examinations. This was done to compare the RDs found for the ${ }^{18} \mathrm{~F}-\mathrm{FET}$-avid lesions with the RDs of the 10 reference regions.

\section{RESULTS}

When the DIXON- and BD-based AC approaches were used in the brain tumor scans (groups A and B), noticeable visual misclassification of air by tissue was found in $94 \%, 97 \%$, and $97 \%$ of the AC maps in the sinus, paranasal sinuses, and ear region, respectively (Fig. 1). For UTE-based AC, visual susceptibility artifacts were noticed in $89 \%, 44 \%$, and $89 \%$ of the AC maps, respectively (Fig. 1). 
TABLE 1

Mean RDs in Percentage ( \pm SD) of All Investigated Values for ${ }^{18}$ F-FET and ${ }^{68}$ Ga-DOTANOC Scans

\begin{tabular}{|c|c|c|c|c|c|c|}
\hline \multirow[b]{2}{*}{ Parameter } & \multicolumn{3}{|c|}{${ }^{18} \mathrm{~F}-\mathrm{FET}$} & \multicolumn{3}{|c|}{${ }^{68} \mathrm{Ga}-\mathrm{DOTANOC}$} \\
\hline & Dixon & UTE & BD & Dixon & UTE & BD \\
\hline $\mathrm{SUV}_{\max }$ & $-10 \pm 3$ & $-4 \pm 3$ & $-3 \pm 3$ & $-11 \pm 8$ & $-11 \pm 8$ & $-3 \pm 6$ \\
\hline$S U V_{\text {mean }}$ & $-10 \pm 4$ & $-4 \pm 3$ & $-3 \pm 3$ & $-11 \pm 7$ & $-11 \pm 8$ & $-3 \pm 5$ \\
\hline Volume & $+1 \pm 9$ & $+2 \pm 8$ & $+7 \pm 28^{\star}$ & $+1 \pm 4$ & $-4 \pm 12$ & $-3 \pm 10$ \\
\hline $\mathrm{LBR}_{\max }$ & $-2 \pm 3$ & $0 \pm 3$ & $0 \pm 5$ & - & - & - \\
\hline $\mathrm{LBR}_{\text {mean }}$ & $-3 \pm 3$ & $0 \pm 3$ & $0 \pm 2$ & - & - & - \\
\hline
\end{tabular}

The clinical reporting of the PET data reconstructed with the reference standard CTref revealed 6 primary and 17 recurrent tumors, 8 PET-negative results $\left({ }^{18} \mathrm{~F}-\mathrm{FET}\right)$, and 11 meningeal tumor formations in the $7{ }^{68} \mathrm{Ga}$-DOTANOC scans. The reporting and diagnosis did not change from CTref using different MR-AC methods for any of these patients. An example for ${ }^{18} \mathrm{~F}$-FET and ${ }^{68} \mathrm{Ga}$-DOTANOC is shown in Figure 2.

\section{Group A}

For the quantitative analysis of the ${ }^{18} \mathrm{~F}$-FET scans, 16 of 31 patients were included. Of the remaining 15 patients, 8 did not have an ${ }^{18} \mathrm{~F}$ FET-avid lesion and 7 patients presented with an ${ }^{18} \mathrm{~F}$-FET uptake in the lesion that was too low for a proper segmentation using the $70 \%$ threshold in the CTref-based PET images. Average RDs of the $\mathrm{VOI}_{70} \mathrm{~S}$ were $+1 \%,+2 \%$, and $+7 \%$ (median, $+2 \%$ ) for DIXON, UTE, and $\mathrm{BD}$, respectively. In 3 datasets (1 patient: DIXON- and BD-based PET, 1 patient UTE-based PET), the $70 \%$ threshold segmentation included the whole brain, and therefore these 3 image series were

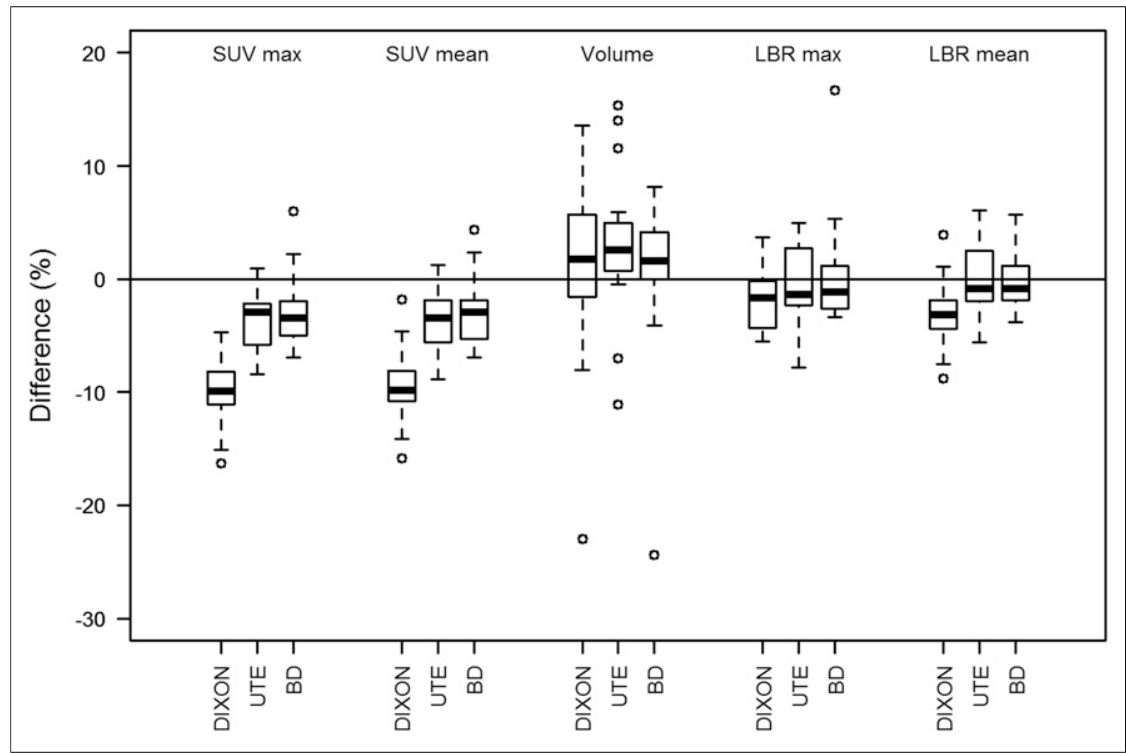

FIGURE 3. Box plots of RD of SUV $V_{\max }$ and $S U V_{\text {mean }}, V \mathrm{VI}_{70}$ (volume), and LBRs of SUV $\mathrm{Smax}_{\text {max }}$ $\left(\mathrm{LBR}_{\text {max }}\right)$ and $\mathrm{SUV} \mathrm{V}_{\text {mean }}\left(\mathrm{LBR}_{\text {mean }}\right)$ according to CTref for ${ }^{18} \mathrm{~F}-\mathrm{FET}$-avid lesions and 3 different AC methods. excluded from the calculations. For the remaining reconstructions, the maximum $\mathrm{RD}$ was found for $\mathrm{BD}$, with the volume expanding to $203 \%$ of the original volume for 1 of 16 patients. A summary of the RDs for $\mathrm{SUV}_{\text {max }}$ and $\mathrm{SUV}_{\text {mean }}$ is given in Table 1. LBRs were similar across all AC methods. All findings are summarized in Figure 3 and Table 1.

\section{Group B}

For ${ }^{68} \mathrm{Ga}$-DOTANOC examinations, analysis included 11 tumor formations and in 5 of 7 patients the pituitary gland (in 2/11 cases, the pituitary gland could not be separated from the tumor formations). Average RDs of the $\mathrm{VOI}_{50}$ s were similar for all AC methods (Table 1), however, with a noticeable spread (Fig. 4). SUV mean and $\mathrm{SUV}_{\max }$ values showed similar average RDs of $-11 \%$ for DIXON and UTE compared with $-3 \%$ for BD (Fig. 4; Table 1). The analysis of the subgroup of lesions located in the skull base $(n=9)$ revealed average $\mathrm{SUV}_{\text {mean }}$ and $\mathrm{SUV}_{\text {max }}$ deviations of $-6 \%$, $-13 \%$, and $-1 \%$ for DIXON, UTE, and $\mathrm{BD}$, respectively. For lesions attached to the skull cap $(n=7)$, average deviations of $-18 \%,-8 \%$, and $-6 \%$ for $\mathrm{SUV}_{\max }$ and $-17 \%,-7 \%$, and $-4 \%$ for $\mathrm{SUV}_{\text {mean }}$ for DIXON, UTE, and BD, respectively (Fig. 5), were found.

\section{Group C}

For ${ }^{18} \mathrm{~F}-\mathrm{FDG}$ imaging, mean whole-brain bias $\pm \mathrm{SD}$ was $-11 \pm 1 \%,-7 \pm 1 \%$, and $-5 \pm 2 \%$ for the DIXON, UTE, and BD ACbased PET images, respectively. The differences in CTref-based PET for the 10 predefined brain reference regions are depicted in Figure 6. A more detailed analysis consisting of the pixel-vice-calculated average RD and corresponding SD for the ${ }^{18}$ F-FDG imaging can be found in the supplemental materials (available at http://jnm.snmjournals. org). The comparison of the $\mathrm{SUV}_{\text {mean }} \mathrm{RDs}$ of the ${ }^{18} \mathrm{~F}-\mathrm{FET}$-avid lesions and the findings of the regional analysis of the ${ }^{18} \mathrm{~F}-\mathrm{FDG}$ scans can be found in Figure 6. 


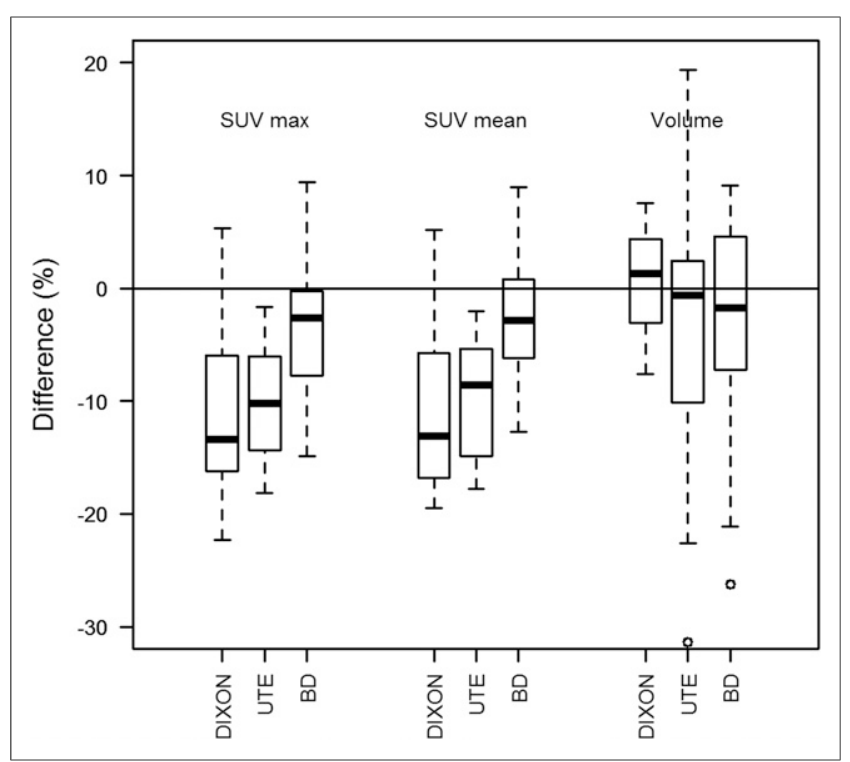

FIGURE 4. Box plots of RD of SUV $V_{\max }$ and $S U V_{\text {mean }}$ and $\mathrm{VOI}_{50}$ (volume) according to CTref for lesions in ${ }^{68} \mathrm{Ga}$-DOTANOC scans and 3 different AC methods. Data included 11 meningeal tumor formations and for 5 patients additionally pituitary gland (in 2 patients, pituitary gland could not be separated from tumor formation).

\section{DISCUSSION}

Standard MR-AC is challenged by pitfalls in MR imaging, such as susceptibility changes leading to signal voids in MR-AC or truncation effects due to the limited transverse field of view $(19,21,29)$. For the DIXON approach, artifacts consisted mainly of artificial filling of air cavities (e.g., the sinuses) with tissue (Fig. 1). Such artifacts were already described by Brendle et al. (30) and are most likely attributed to the properties of the segmentation algorithm, which is part of the DIXON MR-AC. For UTE-based MR-AC, an overestimation of the size of air cavities was present as well as a partial underestimation of bone in the region of the brain (Fig. 1). This known observation (21) is caused by susceptibility artifacts at borders of tissue and air. The consequences of these artifacts are local over- and underestimations of the tracer concentration in and near the affected regions. Examples are the frontal part of the brain or the area around the mastoid cells, for which UTE-based PET images show a drop in activity concentration and DIXON and consequently BD-based PET tend to overcorrect attenuation (Supplemental Figs. 3, 9, 10, and 17).

Despite the differences found, clinical diagnosis based on the visual assessment of the disease after commonly used procedures, including LBRs, did not reveal any differences in diagnostic outcome between the used AC methods. This is attributed to the specificity of the used tracers demonstrating similar local contrast in all cases (Fig. 2) and to the similarity of the LBRs.

For the quantitative evaluation, a threshold-based segmentation approach was used to simulate a clinical scenario when only 1 (predefined, standard) AC method is available. The data presented here show a good agreement of the segmented volumes between the AC methods but with significant deviations in individual cases. This is attributed to the properties of threshold-based segmentation. The segmented volume depends on the value of the maximum pixel and the activity around. The local differences in activity distributions in the PET images reconstructed using different MR-ACs are particularly obvious, for example, near bone (11) and near air cavities, due to the above-described artifacts (Supplemental Figs. 3, 10, and 17 near the frontal sinuses or Supplemental Figs. 2, 9, and 16 near the mastoid cells). The local variations in quantification were reflected in the evaluation of the SUVs. For lesions not near bony structures, as is the case for most of the ${ }^{18} \mathrm{~F}-\mathrm{FET}$ examinations evaluated here, UTE- as well as BDbased PET caused no clinically relevant shift in SUV. In contrast, DIXON-based PET underestimated SUV by $-10 \%$, which is attributed to the lack of bone in the DIXON-based MR-AC (Fig. 3) (11). Nevertheless, for an individual lesion, quantification can differ substantially from the median offset for all AC methods. For the LBR, good accordance was found for all AC methods with a maximum bias spread of $-9 \%$ and $10 \%$. This is expected because of the similarity in bias between hemispheres already shown (23).

For lesions attached to bony tissue, as in the case of the meningeal tumors imaged with ${ }^{68} \mathrm{Ga}$-DOTANOC, BD showed the best comparability with CTref. As expected, the DIXON-based MR-AC underestimated SUVs, which is caused by the lack of bone information in the AC map (11). Of note, UTE-based MRAC did not improve SUV quantification for this kind of lesion compared with DIXONbased MR-AC (Table 1; Fig. 4). For the subgroup of lesions attached to the skull cap, an improvement in SUV quantification could be observed in comparison to DIXON-based PET. Nevertheless, for lesions near the skull base, the highest RDs in SUV of all MR-AC approaches were found for UTE-based AC (Fig. 5). This is most likely attributed to an underestimation of bone and tissue in this region, subsequently leading to an underestimation of attenuation (Fig. 1; for example, mastoid cells, nasal pharynx). Particularly in these cases, BD could improve quantification in comparison to DIXON- and UTE-based MR-AC.

For the whole-brain and regional analysis of the ${ }^{18} \mathrm{~F}-\mathrm{FDG}$ scans, the results of this
FIGURE 5. Box plots of RD of SUV $V_{\max }$ and $S U V_{\text {mean }}$ and $\mathrm{VOI}_{50}$ (volume) according to CTref for evaluated lesions in ${ }^{68} \mathrm{Ga}-\mathrm{DOTANOC}$ scans and 3 different AC methods. (A) Findings for lesions and pituitary gland in skull base $(n=9)$. (B) Results for lesions attached to skull cap $(n=7)$. 


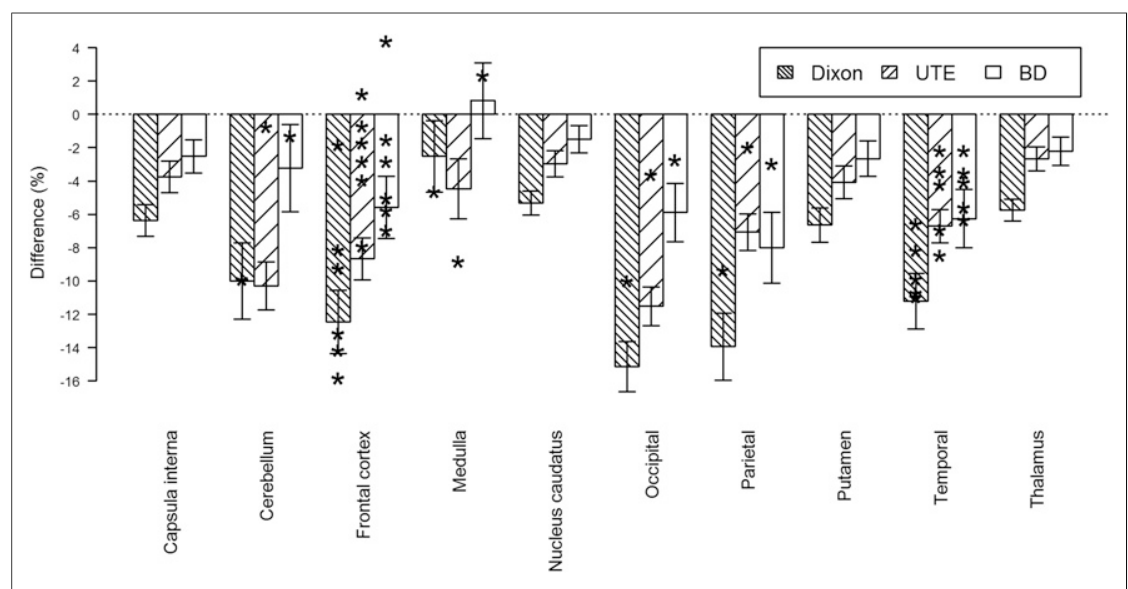

FIGURE 6. Average RD of 10 reference regions within brain for ${ }^{18} \mathrm{~F}-\mathrm{FDG}$ scans of healthy subjects. Error bars correspond to respective RD SD for each reference region. ${ }^{*}$ Corresponding $\mathrm{RDs}$ of $\mathrm{SUV}_{\text {mean }}$ for $\mathrm{VOI}_{70}$ of ${ }^{18} \mathrm{~F}$-FET-avid lesions. Lesions were assigned to reference regions used in ${ }^{18} \mathrm{~F}-\mathrm{FDG}$ evaluation by experienced nuclear medicine expert.

study agree only partly with previous reports in the literature. Although the RD between the regions is similar to that reported in other studies $(21,23,24)$, the absolute bias with respect to the reference standard, CTref, is similar only to the bias reported (24). More specifically, this group reported a whole-brain bias of $-12 \%$ and $-7 \%$ (24) for DIXON and UTE, respectively. These results were similar to findings in our study: $-11 \%$ and $-7 \%$, respectively. However, in another study, Koesters et al. reported a lower whole-brain bias of $-6 \%$ and $2 \%$ for DIXON and BD AC, respectively (23). The difference may likely arise from using the DIXON-based AC as a mask for cropping the CT images to remove the patient table from the $\mathrm{CT}$ data (23) for the purpose of using the CT as a reference standard. The DIXON AC may underestimate the actual extent of the head and, therefore, could result in a smaller representation of the extent of the head in the CTref. To further investigate this difference, we cropped the CTbased AC maps from the ${ }^{18} \mathrm{~F}-\mathrm{FDG}$ imaging using the DIXON MR$\mathrm{AC}$ as a mask, similar to the methodology reported in Koesters et al. (23). Subsequently the PET raw data of the ${ }^{18}$ F-FDG scans were reconstructed again using the cropped $\mathrm{CT}$ attenuation maps. The whole-brain bias was then recalculated as $-6 \%$, and thus similar to the bias reported in Koesters et al. (23). This result reinforces the assumption of a partial underestimation of the extent of the head by the DIXON-based AC, which may lead to an underrepresentation of attenuation in CT-AC when used for cropping the $\mathrm{CT}$ image information for removing the bed from the attenuation data.

In another study evaluating the UTE MR-AC, Aasheim et al. reported a bias of $-3 \%$ for the cerebellum (vs. $10 \%$ in this study) and $+1 \%$ for the putamen $(-4 \%)(21)$. We have currently no explanation for these differences. Perhaps, small deviations in the data processing workflow or the evaluation method may explain these differences. Nevertheless, these observations clearly demonstrate a need for standardization of data processing, analysis, and reporting for the evaluation of AC approaches in PET/ MRI. The choice of a gold standard, in particular, needs to be agreed on to enable a comparison between different studies.

A comparison of the RDs of SUVs of individual lesions and the regional analysis of RD in the ${ }^{18}$ F-FDG scans (Fig. 6) indicates that regional analysis of RDs is not a good predictor for the RD of a lesion. This can be seen in Figure 6, where, depending on the AC method used, between $53 \%$ and $87 \%$ of the lesion's SUV RD is not within $1 \mathrm{SD}$ of the respective regional $\mathrm{RD}$.

\section{CONCLUSION}

Diagnosis of brain tumors in PET/MRI did not vary with the MR-AC methods used when based on visual assessment and LBRs. UTE- and BD-based AC are clinically acceptable for SUV calculations of tracer uptake in lesions located within the brain. However, for lesions near bony structures, especially the skull base, only the model-based approach (BD) showed results comparable with the standard $\mathrm{AC}$ method (CTref). Nevertheless, for the quantification of individual lesions large deviations from CTref can be observed with all MR-AC methods on an individual-lesion basis.

\section{DISCLOSURE}

This study was funded partly by a research grant as part of a Master research agreement between the Medical University of Vienna and the Siemens Healthcare GmbH. Matthias Fenchel is an employee of Siemens. No other potential conflict of interest relevant to this article was reported.

\section{ACKNOWLEDGMENTS}

We thank Martin Lassen, Matthew Difranco, and Jacobo CalGonzalez for their helpful advice concerning data processing. Further, we thank the technologist teams operating the PET/MRI and the PET/CT systems. We are grateful to Neydher Berroteran and his colleagues for the production of the tracers.

\section{REFERENCES}

1. Ellingson BM, Bendszus M, Boxerman J, et al. Consensus recommendations for a standardized brain tumor imaging protocol in clinical trials. Neuro-oncol. 2015;17:1188-1198.

2. Albert NL, Weller M, Suchorska B, et al. Response assessment in NeuroOncology Working Group and European Association for Neuro-Oncology recommendations for the clinical use of PET imaging in gliomas. Neuro-oncol. 2016;18:1199-1208.

3. Bailey DL, Antoch G, Bartenstein P, et al. Combined PET/MR: the real work has just started-summary report of the third international workshop on PET/MR imaging; February 17-21, 2014, Tübingen, Germany. Mol Imaging Biol. 2015;17:297-312.

4. Bailey DL, Barthel H, Beuthin-Baumann B, et al. Combined PET/MR: where are we now? Summary report of the second international workshop on PET/ MR imaging April 8-12, 2013, Tubingen, Germany. Mol Imaging Biol. 2014;16:295-310.

5. Bailey DL, Pichler BJ, Gückel B, et al. Combined PET/MRI: multi-modality multi-parametric imaging is here. Mol Imaging Biol. 2015;17:595-608.

6. Varrone A, Asenbaum S, Vander Borght T, et al. EANM procedure guidelines for PET brain imaging using $\left[{ }^{18} \mathrm{~F}\right] \mathrm{FDG}$, version 2. Eur J Nucl Med Mol Imaging. 2009;36:2103-2110.

7. Hellwig S, Amtage F, Kreft A, et al. $\left[{ }^{18} \mathrm{~F}\right]$ FDG-PET is superior to $\left[{ }^{123} \mathrm{I}\right] \mathrm{IBZM}-$ SPECT for the differential diagnosis of parkinsonism. Neurology. 2012;79:13141322 . 
8. Schöll M, Damián A, Engler H. Fluorodeoxyglucose PET in neurology and psychiatry. PET Clin. 2014;9:371-390.

9. Afshar-Oromieh A, Giesel FL, Linhart HG, et al. Detection of cranial meningiomas: comparison of ${ }^{68} \mathrm{Ga}$-DOTATOC PET/CT and contrast-enhanced MRI. Eur J Nucl Med Mol Imaging. 2012;39:1409-1415.

10. Boellaard R, Quick HH. Current image acquisition options in PET/MR. Semin Nucl Med. 2015;45:192-200.

11. Andersen FL, Ladefoged CN, Beyer T, et al. Combined PET/MR imaging in neurology: MR-based attenuation correction implies a strong spatial bias when ignoring bone. Neuroimage. 2014;84:206-216.

12. Meikle SR, Bailey DL, Hooper PK, et al. Simultaneous emission and transmission measurements for attenuation correction in whole-body PET. J Nucl Med. 1995;36:1680-1688.

13. Kinahan PE, Townsend DW, Beyer T, Sashin D. Attenuation correction for a combined 3D PET/CT scanner. Med Phys. 1998;25:2046-2053.

14. Kinahan PE, Hasegawa BH, Beyer T. X-ray-based attenuation correction for positron emission tomography/computed tomography scanners. Semin Nucl Med. 2003;33:166-179.

15. Keereman V, Mollet P, Berker Y, Schulz V, Vandenberghe S. Challenges and current methods for attenuation correction in PET/MR. MAGMA. 2013;26:8198 .

16. Wagenknecht G, Kaiser H-J, Mottaghy FM, Herzog H. MRI for attenuation correction in PET: methods and challenges. MAGMA. 2013;26:99-113.

17. Delso G, Furst S, Jakoby B, et al. Performance measurements of the Siemens mMR integrated whole-body PET/MR scanner. J Nucl Med. 2011;52:19141922.

18. Martinez-Möller A, Souvatzoglou M, Delso G, et al. Tissue classification as a potential approach for attenuation correction in whole-body PET/MRI: evaluation with PET/CT data. J Nucl Med. 2009;50:520-526.

19. Rausch I, Rust P, Difranco MD, et al. Reproducibility of MRI Dixon-based attenuation correction in combined PET/MR with applications for lean body mass estimation. J Nucl Med. 2016;57:1096-1101.
20. Aznar MC, Sersar R, Saabye J, et al. Whole-body PET/MRI: the effect of bone attenuation during MR-based attenuation correction in oncology imaging. Eur J Radiol. 2014;83:1177-1183.

21. Aasheim LB, Karlberg A, Goa PE, et al. PET/MR brain imaging: evaluation of clinical UTE-based attenuation correction. Eur J Nucl Med Mol Imaging. 2015;42:1439-1446.

22. Paulus DH, Quick HH, Geppert C, et al. Whole-body PET/MR imaging: quantitative evaluation of a novel model-based MR attenuation correction method including bone. J Nucl Med. 2015;56:1061-1066.

23. Koesters T, Friedman KP, Fenchel M, et al. Dixon sequence with superimposed model-based bone compartment provides highly accurate PET/MR attenuation correction of the brain. J Nucl Med. 2016;57:918-924.

24. Ladefoged CN, Benoit D, Law I, et al. Region specific optimization of continuous linear attenuation coefficients based on UTE (RESOLUTE): application to PET/MR brain imaging. Phys Med Biol. 2015;60:8047-8065.

25. Carney JPJ, Townsend DW, Rappoport V, Bendriem B. Method for transforming CT images for attenuation correction in PET/CT imaging. Med Phys. 2006;33: 976-983.

26. Pöpperl G, Götz C, Rachinger W, Gildehaus F-J, Tonn J-C, Tatsch K. Value of O(2-[ $\left.{ }^{18} \mathrm{~F}\right]$ fluoroethyl)-1-tyrosine PET for the diagnosis of recurrent glioma. Eur J Nucl Med Mol Imaging. 2004;31:1464-1470.

27. Boellaard R, Krak NC, Hoekstra OS, Lammertsma AA. Effects of noise, image resolution, and ROI definition on the accuracy of standard uptake values: a simulation study. J Nucl Med. 2004;45:1519-1527.

28. Gehler B, Paulsen F, Öksüz MÖ, et al. [ $\left.{ }^{68} \mathrm{Ga}\right]$-DOTATOC-PET/CT for meningioma IMRT treatment planning. Radiat Oncol. 2009;4:56.

29. Keller SH, Holm S, Hansen AE, et al. Image artifacts from MR-based attenuation correction in clinical, whole-body PET/MRI. MAGMA. 2013;26:173-181.

30. Brendle C, Schmidt H, Oergel A, et al. Segmentation-based attenuation correction in positron emission tomography/magnetic resonance. Invest Radiol. 2015;50:339-346. 